\title{
Outcomes of Uncomplicated Type B Intramural Hematoma Patients with Type 2 Diabetes Mellitus
}

\author{
Zhonggui Shan ${ }^{1}$, Qu Chen ${ }^{2}$, Dandan Jiang 3 , Feng Kuang ${ }^{2}$, and Fan Yang ${ }^{2}$ \\ ${ }^{1}$ Teaching Hospital of Fujian Medical University, The First Affiliated Hospital of Xiamen \\ University \\ ${ }^{2}$ The First Affiliated Hospital of Xiamen University \\ ${ }^{3}$ Xinglin Branch of The First Affiliated Hospital of Xiamen University
}

November 19, 2020

\begin{abstract}
Objectives We aimed to summarize the clinical presentations, therapeutic approaches, and outcomes of type B intramural hematoma (IMHB) patients with and without type 2 diabetes mellitus (DM). Methods Patients with uncomplicated IMHBs were included between January 2016 and January 2018 and divided into two groups according to whether or not they had DM. Cox proportional hazard analysis was utilized to investigate the risk factors for aortic-related mortality. Kaplan-Meier survival analysis was used to estimate cumulative mortality and aortic-related mortality. Results A total of 149 patients were included and were divided into the two groups (DM group [ $\mathrm{n}=60]$ and non-DM group [n=89]). Patients in the non-DM group underwent thoracic endovascular aortic repair treatment more frequently ( $12 \%$ vs $2 \%, \mathrm{P}=0.028)$ and had a higher reintervention rate during the follow-up ( 9 in 81 patients, $11 \%$ vs $2 \%, \mathrm{P}=0.043$ ). There were significant differences between the two groups regarding the aorta-related mortality rate during the acute phase $(9 \%$ vs $0 \%, \mathrm{P}=0.042)$ and the all-cause mortality rate $(22 \%$ vs $7 \%, \mathrm{P}=0.011$ ). Ulcer-like projection (ULP) development (during the acute phase) (hazard ratio [HR], 1.07; 95\% confidence interval [CI], 1.01-1.31, $\mathrm{P}=0.008)$, C-reactive protein (CRP) levels (HR, 1.92; 95\% CI, 1.51-2.49, P<0.001) and MMP-9 levels $(\mathrm{HR}, 16.82 ; 95 \% \mathrm{CI}, 7.52-28.71, \mathrm{P}<0.001)$ were associated with an elevated risk for aorta-related mortality. Conclusions IMHBs without DM are not benign and have a considerably high aortic-related mortality rate. ULP development (during the acute phase), CRP levels and maximum MMP-9 levels are associated with an elevated risk for aorta-related mortality.
\end{abstract}

Title:Outcomes of Uncomplicated Type B Intramural Hematoma Patients with Type 2 Diabetes Mellitus

\section{Authors:}

Qu Chen, MD ${ }^{\text {ae }}$, Dandan Jiang, MD ${ }^{\text {be }}$, Feng Kuang, MD, PhD ${ }^{a}$, Fan Yang, MD ${ }^{c}$, Zhonggui Shan, MD, $\mathrm{PhD}^{\mathrm{d}}$,

a: Department of Cardiovascular Surgery, The First Affiliated Hospital of Xiamen University, Xiamen, Fujian Province, People's Republic of China.

b: Department of Internal Medicine, Xinglin Branch of The First Affiliated Hospital of Xiamen University, Xiamen, Fujian Province, People's Republic of China.

c: Department of Radiology, The First Affiliated Hospital of Xiamen University, Xiamen, Fujian Province, People's Republic of China.

d: Department of Cardiovascular Surgery, Teaching Hospital of Fujian Medical University, The First Affiliated Hospital of Xiamen University.

e: The first two authors contributed equally to this work. 


\section{Email:}

Qu Chen: cmuchenqu@163.com

Dandan Jiang: jddcmu@163.com

Feng Kuang: tysonf@sina.com

Fan Yang: buyiwangdao@126.com

Zhonggui Shan: xmchenqu@163.com

Disclosures: The authors of this manuscript have no relationships with industry to disclose.

Sources of Funding: None.

Date Availability Statement: The raw data required to reproduce these findings cannot be shared at this time as the data also forms part of an ongoing study.

\section{Address for Correspondence}

Zhonggui Shan, MD, PhD

Department of Cardiovascular Surgery, Teaching Hospital of Fujian Medical University, The First Affiliated Hospital of Xiamen University, No. 55 Zhenhai Road, Siming District, Xiamen City, Fujian Province, 36100, People's Republic of China

Tel.: +86 15805908147

Fax: +86 0592-2137601

Email: xmchenqu@163.com

Running Head: Type B Intramural Hematoma with Diabetes Mellitus

Word Coun t: 3075

\section{Abstract}

\section{Objectives}

We aimed to summarize the clinical presentations, therapeutic approaches, and outcomes of type B intramural hematoma (IMHB) patients with and without type 2 diabetes mellitus (DM).

\section{Methods}

Patients with uncomplicated IMHBs were included between January 2016 and January 2018 and divided into two groups according to whether or not they had DM. Cox proportional hazard analysis was utilized to investigate the risk factors for aortic-related mortality. Kaplan-Meier survival analysis was used to estimate cumulative mortality and aortic-related mortality.

\section{Results}

A total of 149 patients were included and were divided into the two groups (DM group [n=60] and non-DM group $[\mathrm{n}=89])$. Patients in the non-DM group underwent thoracic endovascular aortic repair treatment more frequently ( $12 \%$ vs $2 \%, P=0.028)$ and had a higher reintervention rate during the follow-up (9 in 81 patients, $11 \%$ vs $2 \%, P=0.043)$. There were significant differences between the two groups regarding the aorta-related mortality rate during the acute phase $(9 \%$ vs $0 \%, P=0.042)$ and the all-cause mortality rate $(22 \%$ vs $7 \%, P$ $=0.011$ ). Ulcer-like projection (ULP) development (during the acute phase) (hazard ratio [HR], 1.07; $95 \%$ confidence interval [CI], 1.01-1.31, $P=0.008$ ), C-reactive protein (CRP) levels (HR, 1.92; 95\% CI, 1.51-2.49, $P<0.001)$ and MMP-9 levels (HR, 16.82; 95\% CI, 7.52-28.71, $P<0.001)$ were associated with an elevated risk for aorta-related mortality. 


\section{Conclusions}

IMHBs without DM are not benign and have a considerably high aortic-related mortality rate. ULP development (during the acute phase), CRP levels and maximum MMP-9 levels are associated with an elevated risk for aorta-related mortality.

\section{Abbreviations:}

CRP: C-reactive protein

ULP: Ulcer-like projection

IMH: Intramural hematoma

IMHB: Type B intramural hematoma

TEVAR: Thoracic endovascular aortic repair

Key words: IMH, Diabetes Mellitus, Mortality, Outcome

\section{Introduction}

Although the current treatment strategy (wait-and-watch strategy) for type B intramural hematoma (IMHB) is in line with the management of type B dissection, the long-term outcomes of IMHB are not as good as those of type B dissection [1-4] . The cause of this phenomenon is that the evolution of IMHB is highly unpredictable and can vary from complete resolution to abrupt rupture. Schoenhoff et al.[5] reported that $43 \%$ of IMHB patients underwent thoracic endovascular aortic repair (TEVAR) during the first two weeks because of visualization of an entry tear and development of an aneurysm, and $19 \%$ of patients received TEVAR after the acute phase and during the first year. Durham and colleagues also reported that the survival rate of IMHB patients who underwent interventional treatment was only $76 \%$, and only $40 \%$ of patients did not require reintervention

\section{[6]}

The prevalence of diabetes mellitus (DM) in patients requiring cardiac surgery is significantly increasing, and achieving tight perioperative glycemic control in DM patients may decrease perioperative morbidity and improve survival [7] . Current studies have demonstrated a negative correlation between DM and the occurrence of aortic diseases [8-11] . However, previous studies are contradictory in that patients with DM were found to have poorer outcomes after abdominal aortic aneurysm repair [12], whereas mortality and clinical complications in type B aortic dissection patients after TEVAR were significantly reduced in DM patients [13]. In our previous study, we demonstrated that uncomplicated type A IMH patients with DM (receiving the "wait-and-watch strategy" and tight glycemic control) may have lower aorta-related mortality and rates of aorta-related adverse events and reintervention than patients in the non-DM group[14] .

Whether patients with uncomplicated IMHB (receiving the "wait-and-watch strategy") may benefit from tight glycemic control remains unclear. Thus, we hypothesized that among uncomplicated IMHB patients receiving the "wait-and-watch strategy" (combined with tight glucose management), patients with DM (compared with patients without DM) would benefit from such a treatment strategy. In this study, we aimed to summarize the clinical presentations, therapeutic approaches, and outcomes of IMHB patients with and without DM.

\section{Methods}

\section{Patient Characteristics}

Individual informed consent was obtained from each patient, and institutional review board approval was obtained from Xiamen University. Patients with uncomplicated IMHBs were included between January 2016 and January 2018 (see the details of the CONSORT diagram in Figure 1 ).

\section{Blood Glucose Management}


Newly diagnosed type 2 DM patients without standard antidiabetic treatments before the onset of IMHB were included. The target blood glucose level included proper blood glucose levels during fasting and premeal states $(80-130 \mathrm{mg} / \mathrm{dL}$ ) and the postprandial state (less than $180 \mathrm{mg} / \mathrm{dL}$ ) [7] . After TEVAR or open surgery, patients with persistently elevated serum glucose levels $(>180 \mathrm{mg} / \mathrm{dL})$ received continuous intravenous insulin perfusion to maintain a serum glucose level $<180 \mathrm{mg} / \mathrm{dL}$ during their stay in the intensive care unit [7] .

\section{Treatment Strategy for Intramural Hematoma}

Patients with uncomplicated IMHBs received medical therapy to control their pain (intravenous opiate analgesia), heart rate (less than 80 beats per minute), and blood pressure (systolic blood pressure between 100 and $120 \mathrm{mmHg}$ ) [1]. The acute phase was defined as the first fourteen days from the onset of IMHB, and the disease was considered stable after fourteen uneventful days (patients were discharged) [1] . Electrocardiographic-triggered CTA (GE Healthcare, Milwaukee, WI) was performed at least twice during the acute phase (once upon admission and once before discharge) in patients without disease progression and in those with well-controlled blood pressure, heart rate and pain; in eventful cases (uncontrollable back pain combined with hemodynamic instability), CTA examinations were adjusted accordingly [1] . The patients were followed clinically according to standard surveillance protocols [1] [15-17] . During the follow-up, CTA was also performed at 1, 3, 6, and 12 months and then annually during the extended follow-up. Disease progression was defined as increased pleural effusion, hematoma thickening (thickness [?]10 mm), development of an aortic pseudoaneurysm, aortic dissection or signs of aortic rupture. Under the following situations, patients would receive TEVAR or surgery treatment: during the acute phase, expansion of IMH and development of pseudoaneurysms despite medical therapy, development of ulcer-like projections (ULP), and signs of aortic rupture (uncontrollable back pain combined with a precipitous decrease in blood pressure); and during the chronic phase, development of aortic dissection, rapid growth of ULPs or aorta diameters $(>5$ $\mathrm{mm} /$ year), maximum aorta diameters $>55 \mathrm{~mm}$, and signs of aortic rupture [1] .

\section{Detection of Serum Matrix Metalloproteinase-9}

Matrix metalloproteinase-9 (MMP-9) is an important diagnostic biomarker in aortic pathophysiology, and MMP-9 can weaken the aortic media by degrading multiple extracellular components; DM patients have a 2-fold decreased level of MMP-9, which can restrict degradation of the aortic wall [16] . Plasma MMP-9 was measured by using an enzyme-linked immunosorbent assay (ELISA). The MMP-9 level was measured on admission, at day 14 (after the acute phase) and day 90 (after the subacute phase), at 6 and 12 months and then annually during follow-up.

\section{Follow-up and Study Endpoints}

All patients were followed for at least three years. The remodeling process was confirmed by the latest CTA scan. The definition of IMHB evolution in the chronic phase included hematoma stability, resolution and worsening. The primary outcome was aortic-related mortality (confirmed by autopsy or CTA examination), and the secondary outcomes were all-cause mortality and aortic remodeling.

\section{Statistical Analysis}

Comparisons between groups were performed by the $t$-test or Mann-Whitney $\mathrm{U}$ test when necessary. Categorical variables are expressed as frequencies and percentages and were compared with the $\chi^{2}$ test and Fisher's exact test when appropriate. A Cox proportional hazard analysis was used to calculate the hazard ratios (HRs) and investigate the risk factors for aorta-related mortality. In the multivariate Cox proportional hazard models, the entry and removal criteria were set at $P=0.05$ and $P=0.20$. Kaplan-Meier survival analysis with the log-rank test was used to estimate the cumulative mortality and aortic-related mortality in different groups. We used the Kolmogorov-Smirnov test to evaluate the normality of the continuous variables. Differences with $P$ values $<0.05$ were considered statistically significant. IBM SPSS for Windows version 26.0 (IBM Corp, Armonk, New York) was used for statistical analysis.

\section{Results}




\section{Patient Characteristics}

After excluding patients with incomplete follow-up data, 149 patients remained, and their data were analyzed (Figure 1 ). These patients were classified into two groups (DM group [n=60] and non-DM group [n=89]) according to whether or not they had DM. The median follow-up times for each group were as follows: DM group (38.0 months, 95\% confidence interval [CI], 36.8-39.1 months) and non-DM group (39.0 months, 95\% CI, 36.7-41.2 months). Compared to those without DM, patients with DM had a higher hypertension rate ( $72 \%$ vs $53 \%, P=0.021)$ as well as lower CRP levels $(5.7 \pm 1.5$ vs $11.3 \pm 2.9 \mathrm{mg} / \mathrm{dL}, P<0.001)$ and white blood cell counts $\left(14.2 \pm 1.9\right.$ vs $\left.18.3 \pm 3.710^{\wedge} 9 / \mathrm{L}, P<0.001\right)$. The geometric measurements of the aorta indicated that patients in the non-DM group had larger ascending aortas $(39.9 \pm 3.8$ vs $37.9 \pm 2.5 \mathrm{~mm}, P<0.001)$ and descending aortas $(37.9 \pm 3.5$ vs $34.9 \pm 2.8 \mathrm{~mm}, P<0.001)$ as well as thicker hematomas $(9.5 \pm 1.2$ vs $7.0 \pm 1.3$ $\mathrm{mm}, P<0.001)$ than those in the DM group. The other demographic characteristics, such as concomitant diseases and medical treatment strategy, were similar between the two groups (Table 1 ). The MMP-9 levels in the DM group were lower than those in the non-DM group at each timepoint $(P<0.001$, Figure 2$)$.

\section{Disease Progression, Treatment and Follow-up}

During the acute phase, the occurrence rate of disease progression (20 of 89 patients) was higher in the non-DM group than in the DM group (4 of 60 patients) ( $22 \%$ vs $7 \%, P=0.011$ ), and disease progression occurred significantly earlier in the non-DM group than in the DM group $(6.4 \pm 1.3$ vs $12.0 \pm 1.4$ days, $P$ $<0.001$ ). The incidence of disease progression was greater in non-DM patients than in DM patients, and the types of disease progression are listed in Table 2. Compared to those in the DM group, more patients in the non-DM group received TEVAR treatment during the acute phase $(12 \%$ vs $2 \%, P=0.028)$. The two deaths after TEVAR treatment in the non-DM group included one patient who died of retrograde type A aortic dissection and one patient who died of aortic rupture (Figure 2 and Figure 3 ).

The aorta-related mortality in patients with IMHBs is also summarized inTable $\mathbf{2}$ and Figure $\mathbf{4}$. There were significant differences in aorta-related death among the DM and non-DM groups during the acute phase $(<14$ days) ( $0 \%$ vs $9 \%, P=0.042$ ) (Table 2 ). The non-DM group also had a higher reintervention rate during follow-up than the DM group (9 in 81 patients, $11 \%$ vs $2 \%, P=0.043$ ). Additionally, the non-DM group had a higher probability of hematoma worsening $(22 \%$ vs $5 \%, P=0.004)$ and a higher rate of aorta-related mortality during the follow-up period ( $11 \%$ vs $2 \%, P=0.043)$ than the DM group (Table 2 ).

The Cox regression analysis revealed that ULP development (during the acute phase) (HR, 1.07; 95\% CI, 1.01-1.31, $\mathrm{P}=0.008)$, CRP level (HR, 1.92; 95\% CI, 1.51-2.49, $\mathrm{P}<0.001)$ and MMP-9 level (HR, 16.82; 95\% CI, 7.52-28.71, $\mathrm{P}<0.001$ ) were associated with an elevated risk for aorta-related mortality (Table 3 ). The Kaplan-Meier survival analysis revealed a significant increase in the aortic-related mortality rate in the DM group compared with that in the non-DM group $(P=0.0023$, Figure 4$)$.

\section{Discussion}

IMH is part of the spectrum of acute aortic syndromes (5-25\%) and is more common in the descending thoracic aorta (type B, 60-70\%) than in the ascending aorta [1-3]. IMH without malperfusion syndrome is always regarded as a benign disease, and the "wait-and-watch strategy" (initial medical treatment plus necessary thoracic endovascular aortic repair treatment) is the first recommendation for these patients [13][17]. Many potential risk factors for IMHB have also been summarized, including a maximum aorta diameter larger than $45 \mathrm{~mm}$, increased pleural effusion, hematoma thickening larger than $10 \mathrm{~mm}$, ULP development, and elevated CRP levels [1]. Overall, patients with IMHB have a more favorable long-term prognosis than patients with aortic dissection and an in-hospital mortality risk lower than $10 \%$ during the acute phase[1]. The mortality rate after TEVAR during the acute phase in our study was $18 \%$ in the nonDM group (Table 2 ), similar to the report by Schoenhoff et al. (the mortality rates of TEVAR within and after the first two weeks were $25 \%$ and $29 \%$, respectively) and was higher than that of type B aortic dissection patients who underwent TEVAR treatment (0\% to 18\%, and median 6\%) [5] . Falconi et al. [18] reported that of 27 type B IMHs in patients managed conservatively and followed for a mean of 33 months, $47 \%$ underwent regression, $14 \%$ remained stable, and 39\% progressed to aortic dissection or enlargement. 
Motoyoshi et al. [18]reported that of 26 patients managed medically, 6 patients (23\%) had spontaneous regression, and 7 patients $(27 \%)$ required a surgical procedure. Although the non-DM group in our study had a higher reintervention rate than the DM group during the follow-up $(11 \%$ vs $2 \%, P=0.044)$, the reinvention rate was obviously lower than that in previous studies $(60 \%$ [6], 39\% [18] , 27\%[19] ).

Newly diagnosed type 2 DM patients with IMHBs probably benefit from antidiabetic treatments, and tight glycemic control may influence IMHB evolution. The potentially protective value of DM has been well described [8-11], and the possible explanations include the increasing matrix of the aortic wall (suppression of plasmin and decreased levels/activity of MMP-9) and decreasing aortic mural macrophage infiltration and neovascularization [16]. MMP-9 is involved in tissue degradation and remodeling in aortic dissection and is significantly increased in aortic dissection patients, and a higher level of MMP-9 can weaken the aortic media by degrading multiple extracellular components; DM patients have a 2-fold decreased level of MMP-9, which may restrict degradation of the aortic wall[16]. Unlike the effect of hyperglycemia on immune cell activity in type $2 \mathrm{DM}$, the impact of insulin deficiency in type $2 \mathrm{DM}$ on macrophage activity has not been widely studied. Tessaro et al. demonstrated that the administration of exogenous insulin in diabetes may enhance the immune activity of macrophages [20], and insulin treatment may diminish this protective effect of hyperglycemia that prevents aortic aneurysm development (under laboratory conditions)[21]. For these reasons, it seems that the administration of insulin is probably a risk factor for disease progression in type 2 DM patients with IMHBs. In our study, after receiving tight glycemic control recommended by guidelines [7], patients in the DM group had an MMP-9 level that was dramatically increased (reached the highest value) during the acute phase, and the MMP-9 level reached the highest level earlier in the DM group than in the non-DM group. However, the MMP-9 levels in the DM group were lower than those in the non-DM group at each timepoint $(P<0.001$, Figure 2$)$, and patients in the non-DM group had a 2-fold higher level of MMP-9 after day 90 than patients in the DM group, which likely indicated that the potentially protective effect of hyperglycemia did not decrease after the administration of insulin, which could likely explain the significantly lower aorta-related mortality in the DM group during the follow-up period. Until now, the mechanism of the protective effect of hyperglycemia and the administration of insulin and other antidiabetic treatments in aortic diseases has remained unclear and requires further study.

For this higher mortality rate in the non-DM group than in the DM group after TEVAR treatment, one possible explanation was the larger aorta diameters and greater hematoma thickness in the non-DM group. Bischoff et al. [22] indicated that patients with larger ascending aorta diameters had a worse clinical outcome than those with smaller aortas. Ye et al. also recommended that patients with aorta diameters larger than $45 \mathrm{~mm}$ and hematoma thicknesses greater than $10 \mathrm{~mm}$ should be included in the "complicated" group, and more aggressive TEVAR treatment was required in these patients to prevent fatal IMHB evolution[23] . In addition, the geometry of the aorta can be influenced by TEVAR, which can result in a poor outcome, especially in those with ascending aortas larger than $40 \mathrm{~mm}$ (who are more likely to suffer from retrograde type A aortic dissection after TEVAR)[24] . In the non-DM group, one patient died of retrograde type A aortic dissection after TEVAR during the acute phase. Both patients who died after TEVAR in the nonDM group had ascending aorta diameters larger than $40 \mathrm{~mm}$ (Supplement 1 ). Thus, an ascending aorta diameter larger than $40 \mathrm{~mm}$ is another potential evolutionary predictor of IMHB.

The other possible explanation for this higher mortality rate in the non-DM group is the improper timing of TEVAR and the stent graft size. The current timing of TEVAR for uncomplicated IMHB is in line with that for uncomplicated type B aortic dissection (patients with IMH expansion despite medical therapy and ULP development) [1][4] . However, the disease progression of IMHB is highly unpredictable, and the current guidelines are based on good outcomes of TEVAR for type B aortic dissection[25][26] and on some small sample studies for IMHBs[1][4][27][28]. In our study, all 12 patients with uncontrollable symptoms (especially refractory pain) underwent TEVAR. The two patients who finally died of aorta-related complications still complained of refractory pain after TEVAR (Supplement 1 ). Juvonen et al. [29] reported that patients with uncharacteristic or atypical pain had a higher risk of rupture over time than those without pain. Medical management of such patients is unwarranted unless their life expectancy and quality of life are markedly impaired [30] . A more aggressive TEVAR procedure (while the symptoms are 
still controllable) would likely have better outcomes (acute phase mortality: 0\%, Bischoff et al. [22] , 0\%, Ye et al. [23] ), and this more aggressive strategy is likely to prevent the progression of IMHB and result in a better prognosis than medical treatment plus necessary TEVAR (for patients with uncontrollable symptoms and fatal evolution). Further studies are necessary to evaluate whether patients with uncomplicated IMHBs without diabetes mellitus could benefit from more aggressive TEVAR treatment while the symptoms are still controllable. In addition, in our study, the diameter of the stent graft was oversized by less than $10 \%$, which was smaller than the diameters in the study by Schoenhoff et al. (10\% to 15\%). [5]. However, both in our study and in the study by Schoenhoff et al. [5], the mortality rates were higher than those after TEVAR for type B aortic dissection[4][31] . For aortic dissection, there is a general agreement in the current guidelines that the oversizing factor should not exceed $5 \%$ [31]. Is it necessary to utilize less oversizing (not exceeding $5 \%$ [31] ) for stent grafts similar to the recommendations for aortic dissection in patients with IMHB?

In addition, ULP development during the acute phase and CRP levels were associated with an elevated risk for aorta-related mortality. Moral et al. [32] reported that $10 \%$ of patients with uncomplicated IMHBs suffered from ULP development during the acute phase; among these patients, the disease progression rate was as high as $91 \%$, and $36 \%$ of them died of aortic-related complications. Kitai et al. reported that $71 \%$ of patients with IMHBs had ULPs during the acute phase, of which $76 \%$ showed progression (enlargement or progression to an aortic aneurysm) and required further treatment [33]. The potential risk factors for ULP development are large aorta diameters and hematoma thicknesses, which are also evolutionary predictors of IMHB[30]. In addition, a higher CRP level may be indirect evidence that could represent the degree of inflammation and pathophysiological changes in the aortic wall under such unstable hemodynamic conditions; a sustained, high CRP level has significant prognostic value in IMHB patients [34].

There are several limitations to this study. First, in this study, the majority of patients received only two CTA examinations during the acute phase (once upon admission and once before discharge), and the diagnosis of ULPs could have been influenced by the preciseness of the CTA images. Closer monitoring for the development of ULPs is necessary in further studies. Second, in future studies, we should enroll more patients with different medical treatment strategies, which may provide more meaningful insight into the influence of these strategies on IMHBs.

\section{Summary}

Our study suggests that IMHB without diabetes mellitus is not a benign disease and has considerably high aortic-related mortality rates both in the acute phase and during long-term follow-up. Furthermore, ulcer-like projection development (during the acute phase), C-reactive protein levels and maximum matrix metalloproteinase-9 levels are associated with an increased risk of aorta-related mortality.

\section{Acknowledgments}

The authors thank Dandan Jiang, a good wife and a loving mother of her family, for her contributions to the data collection and statistical review and for providing emotional support to Dr. Qu Chen.

\section{Figure 1 CONSORT Diagram of Patient Selection}

The CONSORT diagram of the patient selection process is illustrated in this figure. The definition of "uncomplicated" was based on the status of the syndrome after medical treatment (without signs of aortic rupture on admission and well controlled chest/back pain) and the radiographic findings from computed tomography angiography examinations upon admission (without periaortic hematoma or ulcer-like projection) [1][4]. Eligible uncomplicated type B IMH patients who refused further medical treatments and those without complete imaging records, laboratory test results and follow-up data were considered missing data. Patients who refused further treatment, who had incomplete imaging, etc., were excluded.

\section{Figure 2 Levels of Plasma Matrix Metalloproteinase-9 in the Two Groups}

Plasma matrix metalloproteinase-9 (MMP-9) was measured by using an enzyme-linked immunosorbent assay. The levels of MMP-9 were measured on day 14 (after the acute phase), on day 90 (after the subacute phase), 
at 6 and 12 months and then annually during the follow-up. The MMP-9 levels in the DM group were lower than those in the non-DM group at each timepoint $(P<0.001)$.

\section{Figure 3 Evolution of Type B Intramural Hematoma in the non-DM Group}

A)This patient with diabetes mellitus (DM) suffered from hematoma thickening.

B) This patient with ulcer-like projection (ULP) development received emergency thoracic endovascular aortic repair (TEVAR).

C) This patient had increased pleural effusion.

D) This patient with a ULP and increased pleural effusion underwent TEVAR because of aortic rupture signs and died of a retrograde type A aortic dissection.

E) The rapid growth of a ULP that became an aneurysm during follow-up.

Figure 4 Kaplan-Meier Survival Analysis Results

The Kaplan-Meier survival analysis revealed a significant increase in the aortic-related mortality rate in the DM group compared with that in the non-DM group $(\mathrm{P}=0.0023)$.

\section{Supplement 1Aorta-related Deaths During the Acute Phase}

1. Erbel R, Aboyans V, Boileau C, Bossone E, Bartolomeo RD, Eggebrecht H, et al. ESC Committee for Practice Guidelines. 2014 ESC Guidelines on the diagnosis and treatment of aortic diseases: document covering acute and chronic aortic diseases of the thoracic and abdominal aorta of the adult. The Task Force for the Diagnosis and Treatment of Aortic Diseases of the European Society of Cardiology (ESC). Eur Heart J 2014; 35:2873-2926.

2. Song JK, Yim JH, Ahn JM, Kim DH, Kang JW, Lee TY, et al. Outcomes of patients with acute type A aortic intramural hematoma. Circulation 2009;120:2046-2052.

3. Pelzel JM, Braverman AC, Hirsch AT, Harris KM. International heterogeneity in diagnostic frequency and clinical outcomes of ascending aortic intramural hematoma. J Am Soc Echocardiogr 2007;20:12601268 .

4. Hiratzka LF, Bakris GL, Beckman JA, Bersin RM, Carr VF, Casey Jr DE, et al. 2010 AC$\mathrm{CF} / \mathrm{AHA} / \mathrm{AATS} / \mathrm{ACR} / \mathrm{ASA} / \mathrm{SCA} / \mathrm{SCAI} / \mathrm{SIR} / \mathrm{STS} / \mathrm{SVM}$ guidelines for the diagnosis and management of patients with thoracic aortic disease. Circulation 2010;121:e266e369.

5. Schoenhoff FS, Zanchin C, Czerny M, Makaloski V, Gahl B, Carrel T, et al. Aorta Related and All-cause Mortality in Patients with Aortic Intramural Haematoma. Eur J Vasc Endovasc Surg. 2017;54:447-453.

6. Durham CA, Cambria RP, Wang LJ, Ergul EA, Aranson NJ, Patel VI, et al. The natural history of medically managed acute type B aortic dissection. J Vasc Surg 2017;61:1192e8.

7. Cosentino F, Grant PJ, Aboyans V, Bailey CJ, Ceriello A, Delgado V, et al. 2019 ESC Guidelines on diabetes, pre-diabetes, and cardiovascular diseases developed in collaboration with the EASD. Eur Heart J. 2019;1-69.

8. Nienaber CA. Diabetes mellitus and thoracic aortic disease: are people with diabetes mellitus protected from acute aortic dissection? J Am Heart Assoc. 2012;1:e001404.

9. D'cruz RT, Wee IJY, Syn NL, Choong AMTL. The association between diabetes and thoracic aortic aneurysms. J Vasc Surg. 2019;69: 263-268.e1.

10. Takagi H, Umemoto T; ALICE (All-Literature Investigation of Cardiovascular Evidence) Group. Negative Association of Diabetes with Thoracic Aortic Dissection and Aneurysm. Angiology. 2017;68: 216-224.

11. Tsai CL, Lin CL, Wu YY, Shieh DC, Sung FC, Kao CH. Advanced complicated diabetes mellitus is associated with a reduced risk of thoracic and abdominal aortic aneurysm rupture: a population-based cohort study. Diabetes Metab Res Rev.2015;31: 190-7.

12. De Rango P, Farchioni L, Fiorucci B, Lenti M. Diabetes and abdominal aortic aneurysms. Eur J Vasc Endovasc Surg. 2014;47: 243-61. 
13. Hongtao Liu, Lei Shi, Tao Zeng, Qingwei Ji, Ying Shi, Ying Huang, et al. Type 2 diabetes mellitus reduces clinical complications and mortality in Stanford type B aortic dissection after thoracic endovascular aortic repair: A 3-year follow-up study. Life Sciences. 2019;230: 104-110.

14. Qu Chen, Dandan Jiang, Feng Kuang, Fan Yang, Zhonggui Shan. Outcomes of type A intramural hematoma: Influence of diabetes mellitus. J Card Surg. 2020;35:1811-1821.

15. Akin I, Kische S, Ince H, Nienaber CA. Indication, timing and results of endovascular treatment of type B dissection.Eur J Vasc Endovasc Surg. 2009;37:289-96.

16. Hsu CY, Su YW, Chen YT, Tsai SH, Chang CC, Li SY, et al. Association between use of oralantidiabetic drugs and the risk of aortic aneurysm: a nested case-control analysis. Cardiovasc Diabetol. 2016;15: 125 .

17. Bossone E, LaBounty TM, Eagle KA. Acute aortic syndromes: diagnosis and management, an update. European Heart Journal 2017;39: 739-49d.

18. Falconi M, Oberti P, Krauss J, Domenech A, Cesáreo V, Bracco D, et al. Different clinical features of aortic intramural hematoma versus dissection involving the descending thoracic aorta. Echocardiography. 2005;22(8):629-635.

19. Motoyoshi N, Moizumi Y, Komatsu T, Tabayashi K. Intramural hematoma and dissection involving ascending aorta. Eur J Cardiothorac Surg. 2003;24(2):237-242.

20. Fernando H G Tessaro, Thais S Ayala, Eduardo L Nolasco, Leonardo M Bella, Joilson O Martins. Insulin Influences LPS-Induced TNF- $\alpha$ and IL-6 Release Through Distinct Pathways in Mouse Macrophages from Different Compartments. Cell Physiol Biochem. 2017;42(5):2093-2104.

21. Miyama N, Dua MM, Yeung JJ, Schultz GM, Asagami T, Sho E, et al. Hyperglycemia limits experimental aortic aneurysm progression. J Vasc Surg. 2010;52: 975-83.

22. Bischoff MS, Meisenbacher K, Wehrmeister M, Böckler D, Kotelis D. Treatment indications for and outcome of endovascular repair of type B intramural aortic hematoma. J Vasc Surg. 2016;64(6):15691579.e2.

23. Ye K, Qin J, Yin M, Jiang M, Li W, Lu X. Acute Intramural Hematoma of the Descending Aorta Treated with Stent Graft Repair Is Associated with a Better Prognosis. J Vasc Interv Radiol. 2017; 28: 1446-1453.

24. Williams JB, Andersen ND, Bhattacharya SD, Scheer E, Piccini JP, McCann RL, et al. Retrograde ascending aortic dissection as an early complication of thoracic endovascular aortic repair. J Vasc Surg. $2012 ; 55: 1255-62$.

25. Nienaber CA, Rousseau H, Eggebrecht H, Kische S, Fattori R, Rehders TC, et al. Randomized comparison of strategies for type B aortic dissection: the investigation of Stent Grafts in Aortic Dissection (INSTEAD) trial. Circulation. 2009;120:2519-28.

26. Nienaber CA, Kische S, Rousseau H, Eggebrecht H, Rehders TC, Kundt G, et al. Endovascular Repair of Type B Aortic Dissection Long-term Results of the Randomized Investigation of Stent Grafts in Aortic Dissection Trial. Circ Cardiovasc Interv. 2013;6:407-16.

27. Li DL, Zhang HK, Cai YY, Jin W, Chen XD, Tian L, et al. Acute type B aortic intramural hematoma: treatment strategy and the role of endovascular repair. J Endovasc Ther. 2010; 17:617-21.

28. Zhang G, Feng Q, Zheng D, Ma L, Li R, Jiang J, Ni Y. Early aggressive medical treatment associated with selective prophylactic aortic stent-grafting for aortic intramural hematoma. Thorac Cardiovasc Surg. 2011; 59:342-8.

29. Juvonen T, Ergin MA, Galla JD, Lansman SL, Nguyen KH, McCullough JN, et al. Prospective study of the natural history of thoracic aortic aneurysms. Ann Thorac Surg. 1997;63:1533-45.

30. Elefteriades JA. Natural history of thoracic aortic aneurysms: indications for surgery, and surgical versus nonsurgical risks. Ann Thorac Surg 2002;74(Suppl):1877-80.

31. Grabenwoger M, Alfonso F, Bachet J, Bonser R, Czerny M, Eggebrecht H, et al. Thoracic Endovascular Aortic Repair (TEVAR) for the treatment of aortic diseases: a position statement from the European Association for Cardio Thoracic Surgery (EACTS) and the European Society of Cardiology (ESC), in collaboration with the European Association of Percutaneous Cardiovascular Interventions (EAPCI). Eur J Cardiothorac Surg 2012;42:17-24. 
32. Moral S, Cuéllar H, Avegliano G, Ballesteros E, Salcedo MT, Ferreira-González I, et al. Clinical implications of focal intimal disruption in patients with type b intramural hematoma. J Am Coll Cardiol. 2017; 69:28-39.

33. Kitai T, Kaji S, Yamamuro A, Kinoshita M, Ehara N, Kobori A, et al. Detection of intimal defect by 64-row multidetector computed tomography in patients with acute aortic intramural hematoma. Circulation. 2011;124(11 Suppl):S174-8.)

34. Kitai T, Kaji S, Kim K, Ehara N, Tani T, Kinoshita M, et al. Prognostic value of sustained elevated C-reactive protein levels in patients with acute aortic intramural hematoma. J Thorac Cardiovasc Surg. 2014;147(1):326-31.

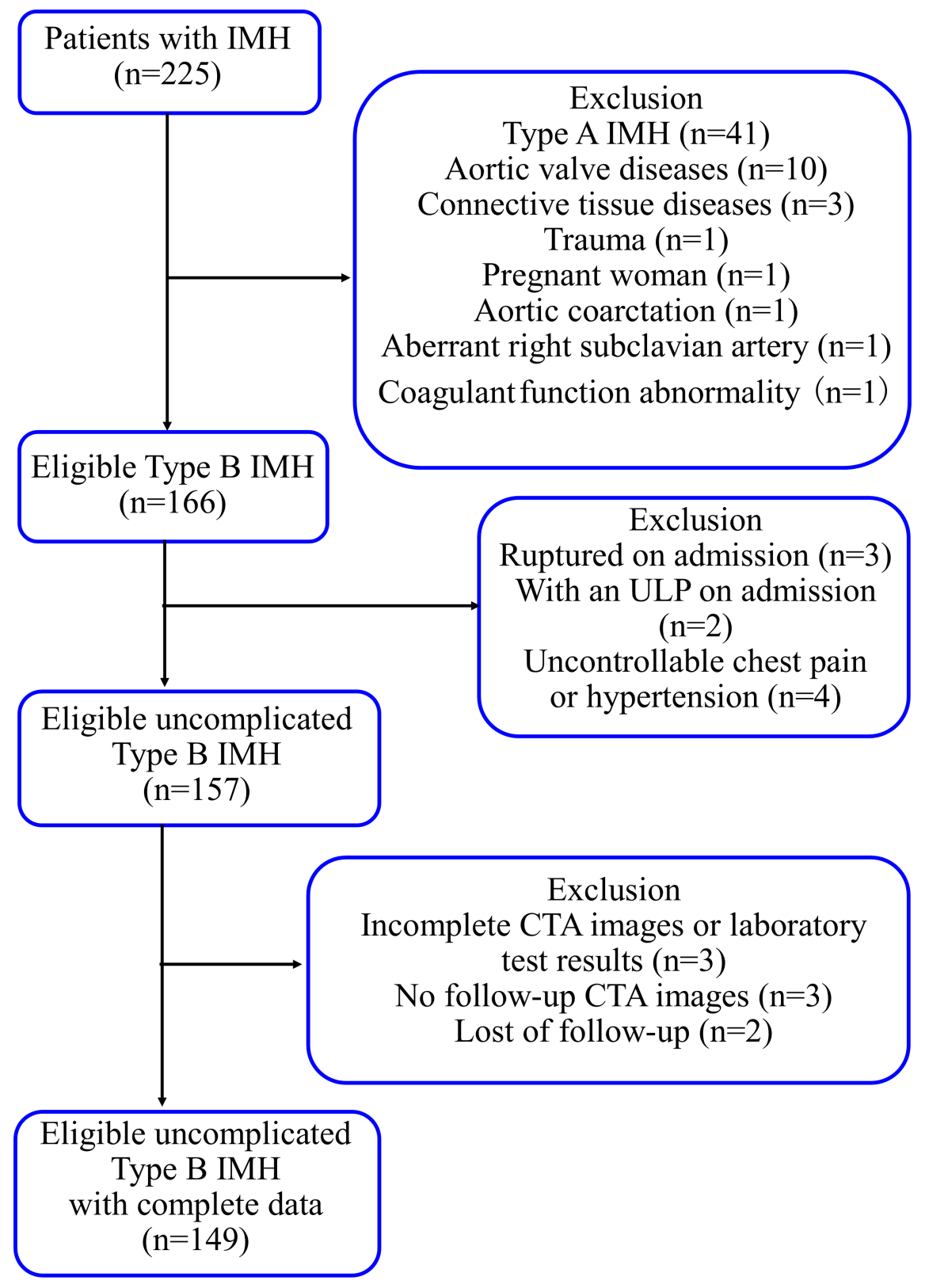


A

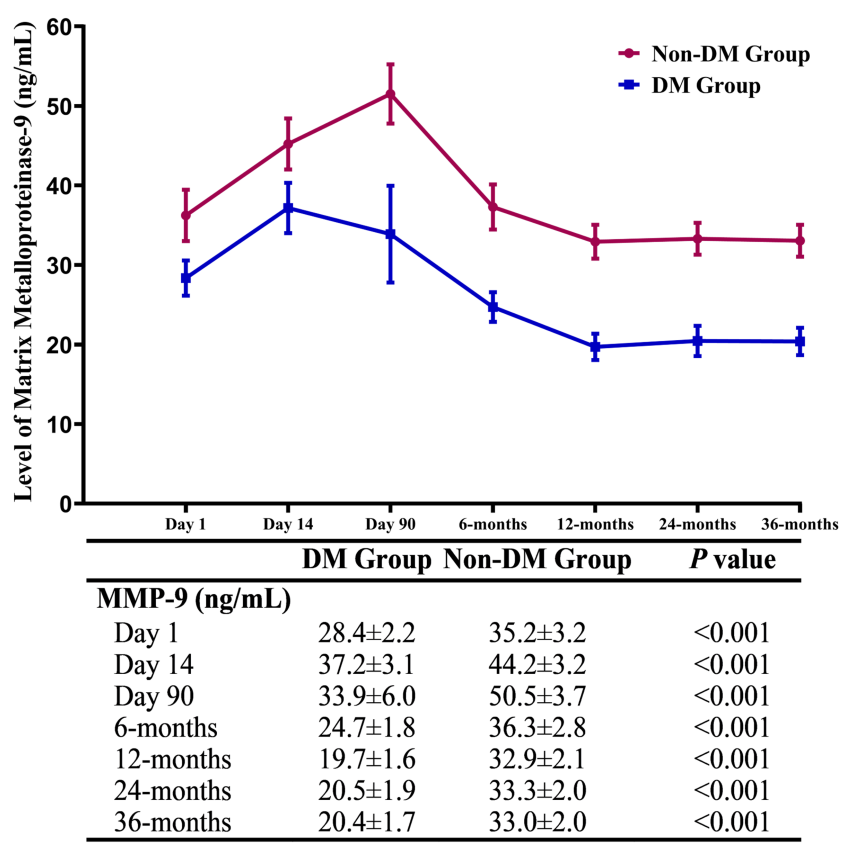

B

B Uncomplicated Type B Intramural Hematoma
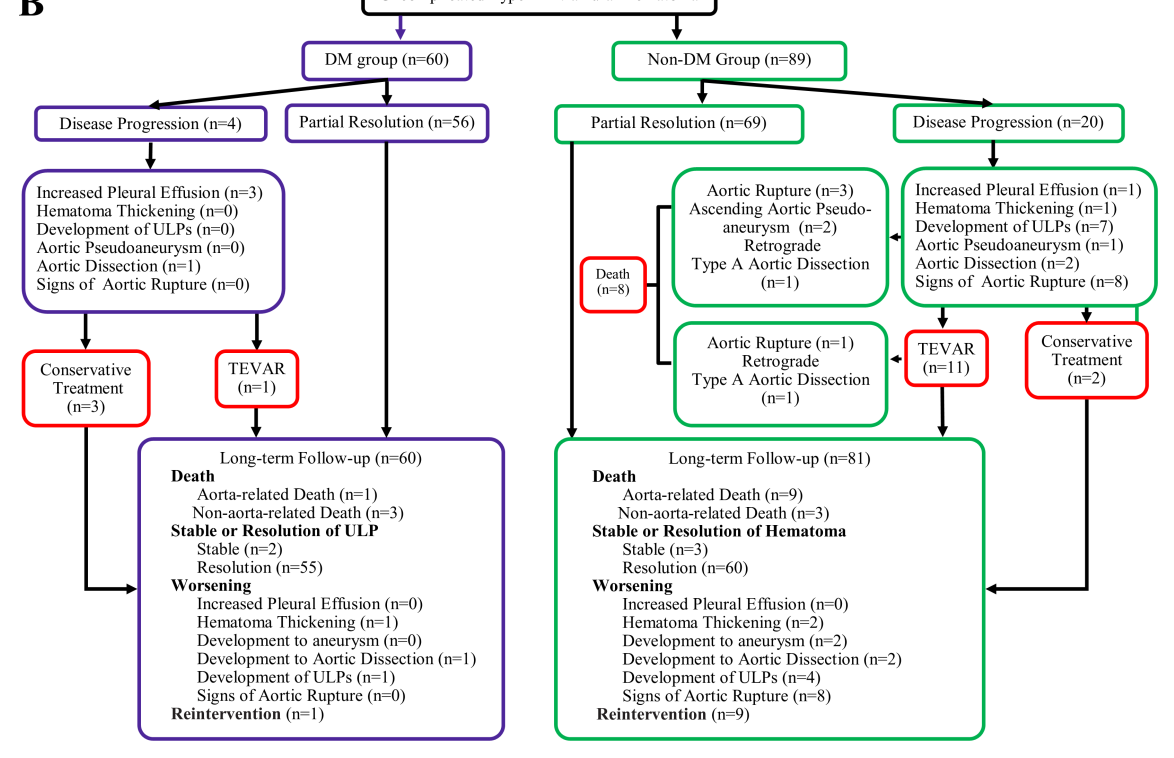

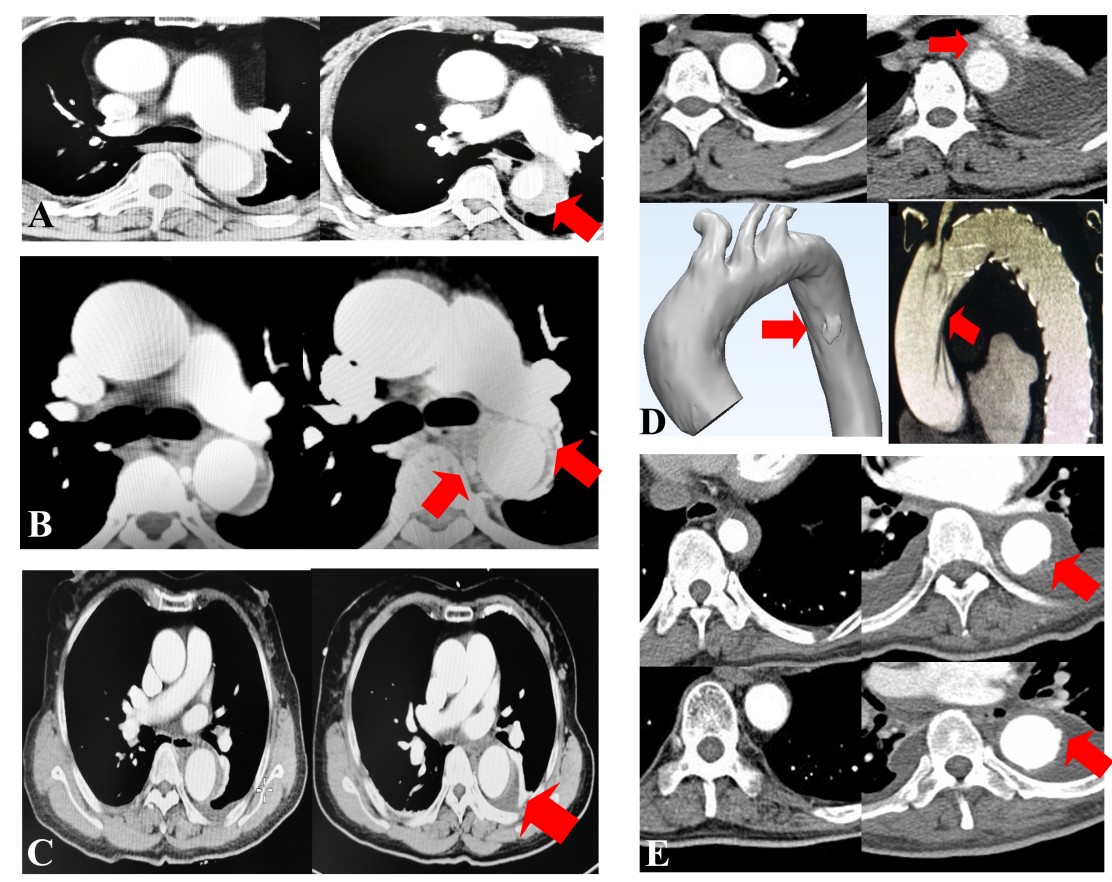

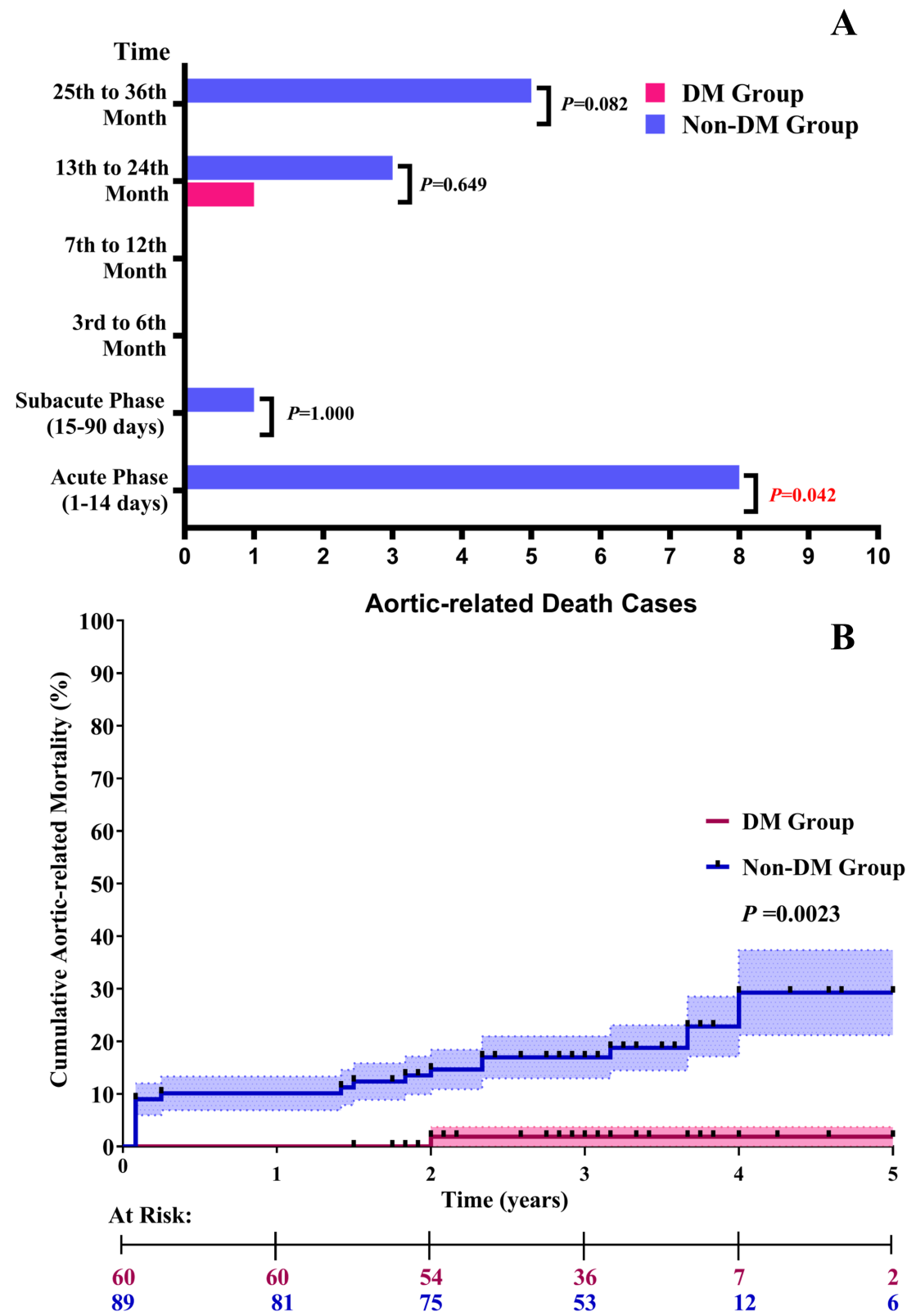

Hosted file

Table 1.pdf available at https://authorea.com/users/316072/articles/493934-outcomes-ofuncomplicated-type-b-intramural-hematoma-patients-with-type-2-diabetes-mellitus

Hosted file

Table 2.pdf available at https://authorea.com/users/316072/articles/493934-outcomes-ofuncomplicated-type-b-intramural-hematoma-patients-with-type-2-diabetes-mellitus

Hosted file

Table 3.pdf available at https://authorea.com/users/316072/articles/493934-outcomes-of- 
uncomplicated-type-b-intramural-hematoma-patients-with-type-2-diabetes-mellitus 